\title{
Les communautés de macrophytes aquatiques comme descripteurs de la qualité de l'eau : exemple de la rivière Moder (Nord-Est France)
}

\author{
G. Thiébaut ${ }^{1}$ \\ S. Muller ${ }^{1}$
}

Mots clés : macrophytes aquatiques, qualité de l'eau, rivière, France.

L'étude phytoécologique de la Moder (rivière du Nord-Est de la France) a été réalisée en 1994 et en 1995 en effectuant des relevés végétaux associés à des analyses physico-chimiques. L'amont, situé sur grès, se caractérise par des eaux peu minéralisées et par un groupement végétal à bryophytes (Amblystegium riparium, A. tenax), Callitriche (C. platycarpa, C. obtusangula, C. hamulata), Ranunculus peltatus, Elodea (E. canadensis, E. nuttallii) et Vaucheria sp. A l'aval, la rivière s'écoule sur substrat marno-calcaire. La minéralisation augmente, une nouvelle espèce d'élodée $E$. ernstae et des algues du genre Cladophora apparaissent. Une comparaison entre les phytocénoses aquatiques de la Moder et celles des rivières phréatiques bicarbonatées calciques du Ried alsacien permet de préciser le rôle des différents paramètres physico-chimiques et en particulier celui de la minéralisation dans la distribution des communautés végétales.

Aquatic macrophyte communities as water quality indicators : example of the river Moder (North-east France)

Keywords : aquatic macrophyte, water quality, river, France.

An assessment of the physico-chemical composition and aquatic macrophyte communities has been carried out during 1994 and 1995 on the river Moder (North-East France). In the upstream area, the river flows on sandstone and is characterized by bryophytes (Amblystegium riparium, A. tenax), Callitriche (C. platycarpa, C. obtusangula, C. hamulata), Ranunculus peltatus, Elodea (E. canadensis, E. nuttallii) and aquatic algae Vaucheria sp. Downstream the river flows on limestone and marl and mineralization increases. Two new macrophytes appear : Elodea ermstae and Cladophora sp. A comparison between the floristic composition of the river Moder with the rivers of the Alsacian Ried allow us to assess the influence of physico-chemical variables, and especially of the mineralization, on the distribution of aquatic macrophyte communities.

\section{Introduction}

L'augmentation de la productivité industrielle et l'intensification des activités humaines sont à l'origine de l'accroissement et de la dispersion de polluants dans les milieux naturels sous forme d'aérosols, de gaz. ou fumées, par infiltration ou par ruissellement. Toutes ces sources de pollutions diffuses ou accidentelles constituent une menace pour les ressources en eau. Aussi, l'étude de la qualité physico-chimique des eaux de surface a-t-elle fait l'objet de nombreux travaux (Carbiener 1990, Lévèque 1994, Moss et al. 1996). La

1. Université de Metz, UPRES «Ecotoxicité, Biodiversité et Santé Environmentale», Laboratoire de Phytoécologie, Ile du Saulcy, 57045 Metz Cedex 01, France. cause première des perturbations relève de «variables d'état», en l'occurrence la disponibilité pour les biocénoses aquatiques de nutriments régulateurs (Barroin 1991) ou la présence d'éléments toxiques dans le milieu: Ce sont ces variables d'état, discriminantes de la qualité de' l'eau, qu'il convient d'étudier en priorité. Leur évolution va définir des processus de modification de la qualité de l'eau (pollution, eutrophisation...).

Capables d'intégrer les perturbations du milieu, les bioindicateurs renseignent sur la qualité des eaux. Les macrophytes aquatiques constituent un compartiment fondamental de l'écosystème aquatique (Hutchinson 1975) et plusieurs indices synthétiques ont été mis au point pour préciser les relations entre les phytocénoses et la qualité des cours d'eau (Harding 1981, Haslam \& Wolseley 1981, Haslam 1987, Newbold \& Holmes 1987, Haury et al. 1996). La majorité des travaux portant sur les relations entre les végétaux aquatiques et la 
qualité de l'eau concernent des rivières minéralisées ou des hydrosystèmes fluviaux (Kohler et al. 1973, Wiegleb 1981, Bornette \& Amoros 1991, Trémolières et al. 1994, Carbiener et al. 1996). Les travaux sur les communautés de macrophytes des cours d'eau faiblement minéralisés restent encore peu nombreux (Haury 1985, Muller 1990, Thiébaut \& Muller 1996).

L'objectif de ce travail est d'analyser les relations entre les groupements végétaux et la composition physico-chimique dans une rivière à minéralisation croissante de l'amont vers l'aval, la Moder. Une comparaison avec les rivières phréatiques du Ried alsacien a pour but de préciser le rôle des différents paramètres physico-chimiques impliqués dans la distribution des groupements végétaux.

\section{Milieux et méthodes d'étude}

\subsection{Le secteur d'étude}

Le secteur d'étude est situé dans le Nord-Est de la France. Il correspond au bassin versant de la Moder de la source de la rivière (Vosges du Nord) jusqu'à l'entrée de Haguenau (plaine d'Alsace). Il englobe ses principaux affluents en plaine. A l'amont, la Moder s'écoule en milieu forestier et draine un substrat gréseux. Ensuite, elle traverse le champ de fracture de Saverne et débouche dans la plaine d'Alsace. Elle sillonne une région agricole dont la roche-mère est constituée de marnes et de calcaires.

La Moder prend sa source sur grès bigarré à environ $320 \mathrm{~m}$ d'altitude, traverse les marnes de la plaine d'Alsace, avant de se jeter dans le Rhin. Elle a ainsi parcouru $90 \mathrm{~km}$. Le cours du ruisseau pratiquement rectiligne coule dans une vallée encaissée aux versants raides dans les Vosges du Nord avant de déboucher dans la plaine d'Alsace. La largeur de la rivière atteint 5 à 6 mètres au débouché sur la plaine et une dizaine de mètres à l'aval de la zone d'étude.

Dans.les. Vosges du Nord, les ruisseaux de tête de bassin versant, traversant des tourbières, correspondent à des eaux à vitesse de courant faible. A mi-parcours, le débit augmente $\left(0,3\right.$ à $\left.0,4 \mathrm{~m}^{3} / \mathrm{s}\right)$ pour atteindre 0,5 à $0,6 \mathrm{~m}^{3} / \mathrm{s}$ au débouché dans la plaine (Thiébaut 1997). En plaine d'Alsace, le débit moyen mensuel des affluents de la Moder est de $0,4 \mathrm{~m}^{3} / \mathrm{s}$ et de $1,2 \mathrm{~m}^{3} / \mathrm{s}$ pour la Moder.

Les mesures des précipitations et des températures sur la période 1972-1995 dans les Vosges du Nord indiquent une pluviométrie moyenne annuelle de 950 $\mathrm{mm}$ et une température moyenne annuelle de $8,7^{\circ} \mathrm{C}$ à Mouterhouse. En plaine d'Alsace, la pluviométrie moyenne annuelle est de 768 mm (période 1912-1991) et la température moyenne annuelle de $9,6^{\circ} \mathrm{C}$ (période 1959-1992).

\subsection{Méthodes}

La Moder et ses affluents ont fait l'objet d'un découpage en tronçons abiotiques selon le protocole mis en place dans le cadre du programme Milieux et Végétaux (Peltre \& Léglize 1992). Le tronçon abiotique d'une rivière se définit comme une portion d'un cours d'eau, caractérisé par des paramètres abiotiques homogènes. La détermination des tronçons homogènes s'établit sur la base de quatre variables discriminantes successives - région naturelle, formation géologique, pente et largeur entre berges -. Les activités anthropiques sont superposées au découpage précédent, et mettent en évidence des tronçons ou parties de tronçons plus ou moins perturbés.

Le choix précis des sites a été réalisé sur le terrain. La section végétale étudiée est représentative de la dynamique d'écoulement et de l'éclairement du tronçon. 13 stations ont été retenues (Fig. 1). Les sites situés sur les affluents (M9 à M13) appartiennent au bassin versant de la Moder et prennent leur source sur grès dans les Vosges du Nord comme la Moder elle-même. Les stations les plus en amont, localisées sur la Moder dans les Vosges du Nord (M1, M2, M3, M4), en plaine (M5, M6) et sur les affluents de la Moder en plaine d'Alsace (M10, M11, M12) sont peu profondes et larges. Les stations (M7, M8, M9, M13) atteignent une dizaine de mètres de largeur et $2-3 \mathrm{~m}$ de profondeur.

\subsubsection{Physico-chimie de la Moder}

Le suivi physico-chimique a été réalisé en juin, août et novembre 1994 et en janvier, juin, août et novembre 1995 sur les 13 stations de l'aire d'étude. Les principaux paramètres physico-chimiques mesurés sont :

- $\mathrm{pH}$, conductivité, anions principaux (chlorures, sulfates, nitrates par chromatographie ionique), cations majeurs (par spectrophotométrie NF 90-005 et NF 90019).

- l'azote ammoniacal N-NH${ }_{4}^{+}$(AFNOR : NF 90015) et les orthophosphates (AFNOR : NF T 90-023)

\subsubsection{Composition floristique}

Tous les végétaux aquatiques et subaquatiques (hydrophytes et hélophytes stricts) sont inventoriés sur une longueur minimale de 50 mètres. Les relevés végétaux - (phanérogames, bryophytes et algues filamenteuses - ont été effectués en 1994 et complétés en 1995, sur les 13 stations retenues (M1 à M13), au cours de la période optimale de croissance végétale.

A chaque espèce présente sur la station étudiée est attribué un coefficient d'Abondance-Dominance (AD) selon la méthode phytosociologique de Braun-Blan- 


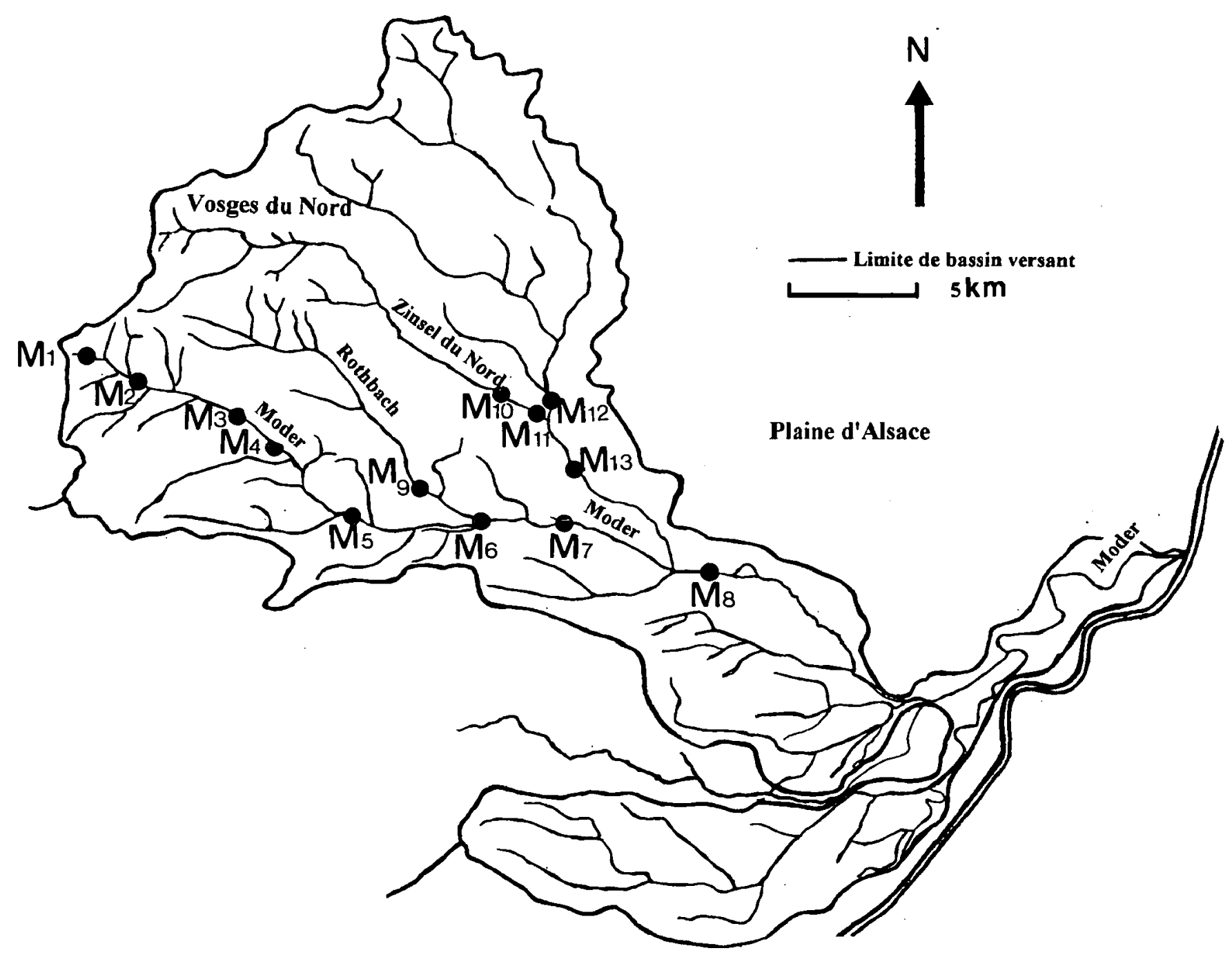

Fig. 1. Carte de localisation des stations d'étude.

Fig. 1. Map showing location of sampling sites.

quet (1964). L'identification des végétaux a été effectuée à l'aide de la «Nouvelle Flore de Belgique, du Grand-Duché de Luxembourg; du Nord de la France et des Régions Voisines» (Lambinon et al. 1992). L'identification des Callitriches a été précisée à l'aide de la clé de détermination de Duvigneaud \&Schotsman (1977). La reconnaissance des algues s'arrête au genre et a été effectuée d'après Bourrelly $(1968,1970$, 1990). L'identification des Bryophytes a été réalisée d'après la flore de Smith (1992).

\subsubsection{Traitement des données}

Une Analyse en Composantes Principales (A.C.P.) normée a été réalisée sur les valeurs moyennes des variables physico-chimiques des 13 stations (Fig. 2). La matrice des résultats contient ainsi 12 variables $(\mathrm{pH}$, conductivité, alcalinité, calcium, magnésium, sodium, potassium, sulfates, chlorures, azote ammoniacal, azote nitrique et orthophosphates) et 13 relevés. Les variables chimiques représentées par les valeurs moyennes stationnelles sont actives. A chaque paramètre physico-chimique correspond ainsi une moyen- ne calculée à partir de 7 valeurs par station ( 3 valeurs en 1994 et 4 valeurs en 1995).

- Une Classification Ascendante Hiérarchique (C.A.H.) a été réalisée sur les 12 valeurs moyennes des paramètres physico-chimiques selon la méthode de Ward et la distance de Pearson au carré.

- Une Analyse Factorielle des Correspondances (A.F.C.) a été effectuée sur 23 espèces d'hydrophytes et d'amphiphytes (phanérogames, bryophytes et algues). La correspondance entre lés espèces floristiques et les variables physico-chimiques a pu être établie par régression linéaire entre le premier axe de l'AFC et le premier axe de l'ACP et entre le deuxième axe de l'AFC et le deuxième axe de l'ACP.

\section{Résultats}

\subsection{Caractéristiques physico-chimiques de la Mo- der}

De l'amont (M1) à l'aval de la Moder (M8), la rivière se minéralise et se charge en nutriments (Tableau 1). 
Tableau 1. Caractéristiques physico-chimiques de la Moder.

Moyennes et écart-types établis sur 7 valeurs (période 1994-1995).

Table 1. Physico-chemical variables of the river Moder.

Mean and standard-deviation values (7 values, in 1994-1995).

\begin{tabular}{|c|c|c|c|c|c|c|c|c|c|c|c|c|}
\hline 64 & $y_{1}$ & owionow & 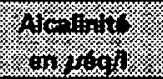 & ( & 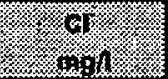 & $\cos ^{2}$ & 4 & mente & (x) & 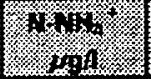 & 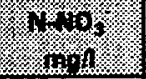 & \% \\
\hline M1 & $7.2+1-0.13$ & $95+1-8$ & $160+/-37$ & $17.3+/-0,8$ & $4.3+1-0,2$ & $9.3+1-0.6$ & $3.6+1-0.2$ & $2.1+1-0.2$ & $2.1+1-0.2$ & $76+1-59$ & $0.9+1-0.1$ & $92+1-33$ \\
\hline M2 & $7.3+1-0.2$ & $130+1-32$ & $260+/-73$ & $15,1+/-2,2$ & $7.3+1-0.5$ & $15,0+1-1,7$ & $4,5+1-0,3$ & $4,4+1-2,9$ & $4,8+/-3.6$ & $154+/-16$ & $1,1+/-0,1$ & $85+/ .16$ \\
\hline M3 & $7.3+/-0.1$ & $106+1-4$ & $615+/ .57$ & $12.9+1-1.8$ & $6.4+1-0.8$ & $10,6+1-2,4$ & $3.4+1-0.2$ & $3.2+1-0.3$ & $3.4+1-0.6$ & $95+1-77$ & $1,1+1-0,2$ & $123+/-45$ \\
\hline M4 & $7,3+1-0,2$ & $109+1.7$ & $616+/-69$ & $12,1+/-1,4$ & $6,5 /-0,6$ & $10.4+/-2.5$ & $3,4+1-0,1$ & $3,8+1-0,6$ & $3,5+1-0,7$ & $442+1-21$ & $1,0+1-0,2$ & $209+1-75$ \\
\hline M5 & $7,6+1-0,2$ & $232+1-25$ & $540+/-108$ & $42,8+1-14,1$ & $8,5+1-0,9$ & $47,5+1-42,4$ & $8,4+1-1,2$ & $5,1+1-0,6$ & $4,1+1-0,3$ & $237+1-14$ & $1,6+1-0,3$ & $303+/-77$ \\
\hline M6 & $7,8+1-0,1$ & $285+1-40$ & $285+1.73$ & $37,9+1.16,7$ & $10,4+1-2,3$ & $38,4+1-7,9$ & $8,7+1-1,6$ & $6,6+1-0,9$ & $3,9+1-0,6$ & $150+1-91$ & $1,7+1-0,7$ & $303+1-69$ \\
\hline M7 & $8,0+1-0,2$ & $274+1-40$ & $760+/-155$ & $42,7+/-11,9$ & $13,2+1-2,0$ & $42,0+1-10,6$ & $8,2+1-1,2$ & $8,1+/-2,1$ & $4,9+1-1,7$ & $160+1-60$ & $2,2+1-0,5$ & $294+1-83$ \\
\hline$M 8$ & $7,9+1-0,2$ & $265+1-32$ & $700+1-136$ & $38,5+1-10,8$ & $16,8+1-3,7$ & $34,4+1-11,0$ & $7,0+1-1,4$ & $9,8+1-2,0$ & $4 ., 3+1-0.8$ & $202+1-93$ & $1.6+1-0,9$ & $277+1-61$ \\
\hline M9 & $7.7+1-0.2$ & $218+1.57$ & $520+1-123$ & $35.3+1-17.2$ & $10,7+1-2,9$ & $48,7+1-42,4$ & $6,3+1-1,8$ & $5,4+1-1,3$ & $4,4+1-0,3$ & $246+1-49$ & $1,8+1-0,7$ & $215+1.45$ \\
\hline $\begin{array}{l}\text { M10 } \\
\text { M11 }\end{array}$ & $\begin{array}{l}7.6+1-0.2 \\
7,7+1-0.3\end{array}$ & $\begin{array}{l}180+1-28 \\
181+1-20\end{array}$ & $\begin{array}{l}440+1-95 \\
400+1-94\end{array}$ & $\begin{array}{l}17.5+1-2.6 \\
20.2+/-4.3\end{array}$ & $\begin{array}{l}17.2+/-6,2 \\
14,8+/-1,4\end{array}$ & $\mid \begin{array}{c}21,6+1-7,6 \\
33,4+1-32,5\end{array}$ & $\begin{array}{r}5.2+1-1,2 \\
10.6+1-13\end{array}$ & $\begin{array}{l}8.1+1-2.9 \\
7.7+1-0.9\end{array}$ & $\begin{array}{l}3,9+1.0,6 \\
4.0+1.0,5\end{array}$ & $\left|\begin{array}{c}219+/-14 \\
113+/-22\end{array}\right|$ & $\begin{array}{l}0,9+1-0,2 \\
0,9+1-0,2\end{array}$ & $\begin{array}{l}161+/-62 \\
147+/-25\end{array}$ \\
\hline M12 & $7,5+1-0,3$ & $153+/-52$ & $400+/-78$ & $15,5+1-4,0$ & $9.1+/-5.0$ & $45.1+1-49.1$ & $5,8+1-3,1$ & $4,9+1-2,8$ & $2,8+1-0,3$ & $66+/-16$ & $0,8+1-0,3$ & $83+1-29$ \\
\hline M13 & $7,6+1-0,2$ & $193+1-20$ & $540+/-94$ & $20,2+1-4,0$ & $14,2+1-2,9$ & $19,8+/-2,1$ & $4,3+1-2,2$ & $7.4+1-0.8$ & $3,4+1-0,7$ & $55+1-27$ & $0,9+1-0,1$ & $120+/-27$ \\
\hline
\end{tabular}

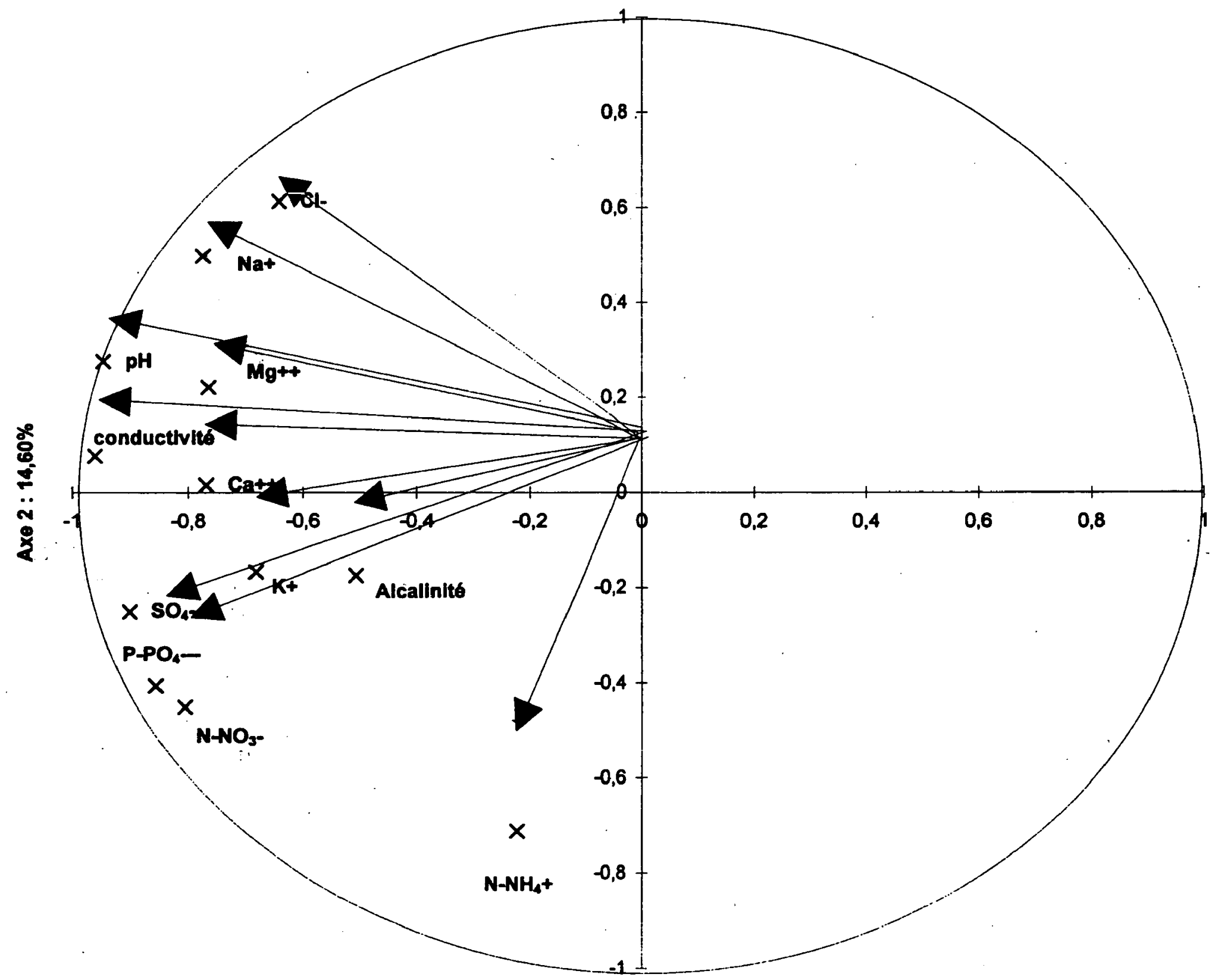

Axe $1: 58,08 \%$

Fig. 2. Analyse en Composantes Principales sur les moyennes des 12 paramètres physico-chimiques. Moyenne établie sur 7 valeurs pour chaque station.

Fig. 2. Principal Component Analysis of the data matrix mean physico-chemical variables $\mathrm{x}$ stations. Mean was calculated from 7 values for each sampling site. 
La minéralisation des eaux sur la partie gréseuse est faible (M1-M4 : conductivité $=95-130 \mu \mathrm{S} / \mathrm{cm}$ ) alors qu'elle augmente fortement en contrebas des collines sous-vosgiennes (M5 : conductivité $=230 \mu \mathrm{S} / \mathrm{cm}$ ). Elle reste relativement stable dans la plaine d'Alsace (M5, M6, M7, M8 : conductivité de 230 à $280 \mu \mathrm{S} / \mathrm{cm}$ ). Le $\mathrm{pH}$ neutre à l'amont $(\mathrm{M} 1: \mathrm{pH}$ moyen $=7,2)$ devient plus alcalin à l'aval (M8: $\mathrm{pH}$ moyen $=8,0)$. De l'amont (M1) vers l'aval (M8), le pouvoir tampon augmente $(M 1$ : alcalinité $=160 \mu$ éq/1 ; M8 : alcalinité =

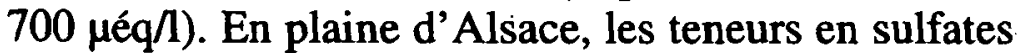
$\left(\left[\mathrm{SO}_{4}{ }^{2-}\right]=40 \mathrm{mg} / \mathrm{l}\right)$ et en calcium $\left(\left[\mathrm{Ca}^{2+}\right]=40 \mathrm{mg} / \mathrm{l}\right)$ sont nettement supérieures à celles mesurées dans les Vosges du Nord ([SO $\left.{ }_{4}{ }^{2-}\right]=15 \mathrm{mg} / 1,\left(\left[\mathrm{Ca}^{2+}\right]=10\right.$ $\mathrm{mg} / \mathrm{l})$. Les eaux sont relativement chargées en nutriment dès l'amont $\left(\mathrm{M} 1:\left[\mathrm{N}-\mathrm{NH}_{4}{ }^{+}\right]=76 \mu \mathrm{g} / \mathrm{l} ;\left[\mathrm{P}^{\left.-\mathrm{PO}_{4}{ }^{3-}\right]}\right.\right.$ $=90 \mu \mathrm{g} / \mathrm{l})$. A l'aval des Vosges du Nord, les teneurs en nutriments atteignent des valeurs élevées (M4 : [N$\left.\left.\mathrm{NH}_{4}{ }^{+}\right]=440 \mu \mathrm{g} / \mathrm{l} ;\left[\mathrm{P}^{-} \mathrm{PO}_{4}{ }^{3-}\right]=210 \mu \mathrm{g} / \mathrm{l}\right)$. En plaine d'Alsace, les teneurs en éléments eutrophisants dans l'eau restent fortes (M8 : $\left[\mathrm{N}^{-\mathrm{NH}_{4}}{ }^{+}\right]=200 \mu \mathrm{g} / \mathrm{l}$; [PPO43- $]=280 \mu \mathrm{g} / 1)$. Les concentrations en azote nitrique augmentent en plaine d'Alsace $\left(\mathrm{M} 7:\left[\mathrm{N}^{-\mathrm{NO}_{3}}{ }^{-}\right]=\right.$ $2,2 \mathrm{mg} / \mathrm{l})$.

Les affluents (M9 à M13) sont moins minéralisés (conductivité moyenne : $190 \mu \mathrm{S} / \mathrm{cm}$ ) et le $\mathrm{pH}$ est plus proche de la neutralité $(\mathrm{pH}$ moyen $=7,6$ ) que la rivière principale (Tableau 1). Ils sont tamponnés (alcalinité : $460 \mu$ éq/l) et renferment des teneurs modérées en sulfates et en calcium $\left(\left[\mathrm{SO}_{4}{ }^{2-}\right]: 20 \mathrm{mg} / 1,\left[\mathrm{Ca}^{2+}\right]: 35\right.$ $\mathrm{mg} / \mathrm{l})$. Le niveau trophique des affluents en plaine d'Alsace varie du mésotrophe pour la Zinsel du Nord $\left(\mathrm{M} 13:\left[\mathrm{N}-\mathrm{NH}_{4}{ }^{+}\right]=55 \mu \mathrm{g} / \mathrm{l} ;\left[\mathrm{P}^{\left.\left.-\mathrm{PO}_{4}{ }^{3-}\right]=120 \mu \mathrm{g} / \mathrm{l}\right) \text { à }}\right.\right.$ l'eutrophe pour le Rothbach (M9: $\left[\mathrm{N}-\mathrm{NH}_{4}{ }^{+}\right]=250$ $\left.\mu \mathrm{g} / \mathrm{l} ;\left[\mathrm{P}_{-}-\mathrm{PO}_{4}{ }^{3-}\right]=215 \mu \mathrm{g} / \mathrm{l}\right)$.

Les trois premières valeurs propres représentent $83,7 \%$ de l'inertie. Le premier axe explique $58,1 \%$ de l'inertie, alors que les deux derniers axes expliquent $14,6 \%$ et $11 \%$ d'inertie respectivement. La première composante principale est corrélée négativement au $\mathrm{pH}(\mathrm{pH} \mathrm{r}=-0,95)$, à la minéralisation (conductivité $\mathrm{r}=$ $-0,96$, calcium $r=-0,77$; magnésium $r=-0,76)$, à l'azote nitrique $(r=-0,80)$ et aux orthophosphates $(r=$ $-0,85)$. La seconde composante principale est corrélée négativement à l'azote ammoniacal $(r=-0,71)$. La troisième composante principale est corrélée négativement à l'alcalinité $(r=-0,61)$.

Les 13 stations ont été classées en fonction de la chimie en 3 groupes par Classification Ascendante Hiérarchique C.A.H. (Fig. 3) :

- le groupe 1 est constitué de 4 stations (M1, M2, M3, M4). Il correspond aux stations amont de la Moder dans les Vosges du Nord. Cet ensemble est caractérisé par des eaux faiblement minéralisées (conductivi-

\section{Similarité}

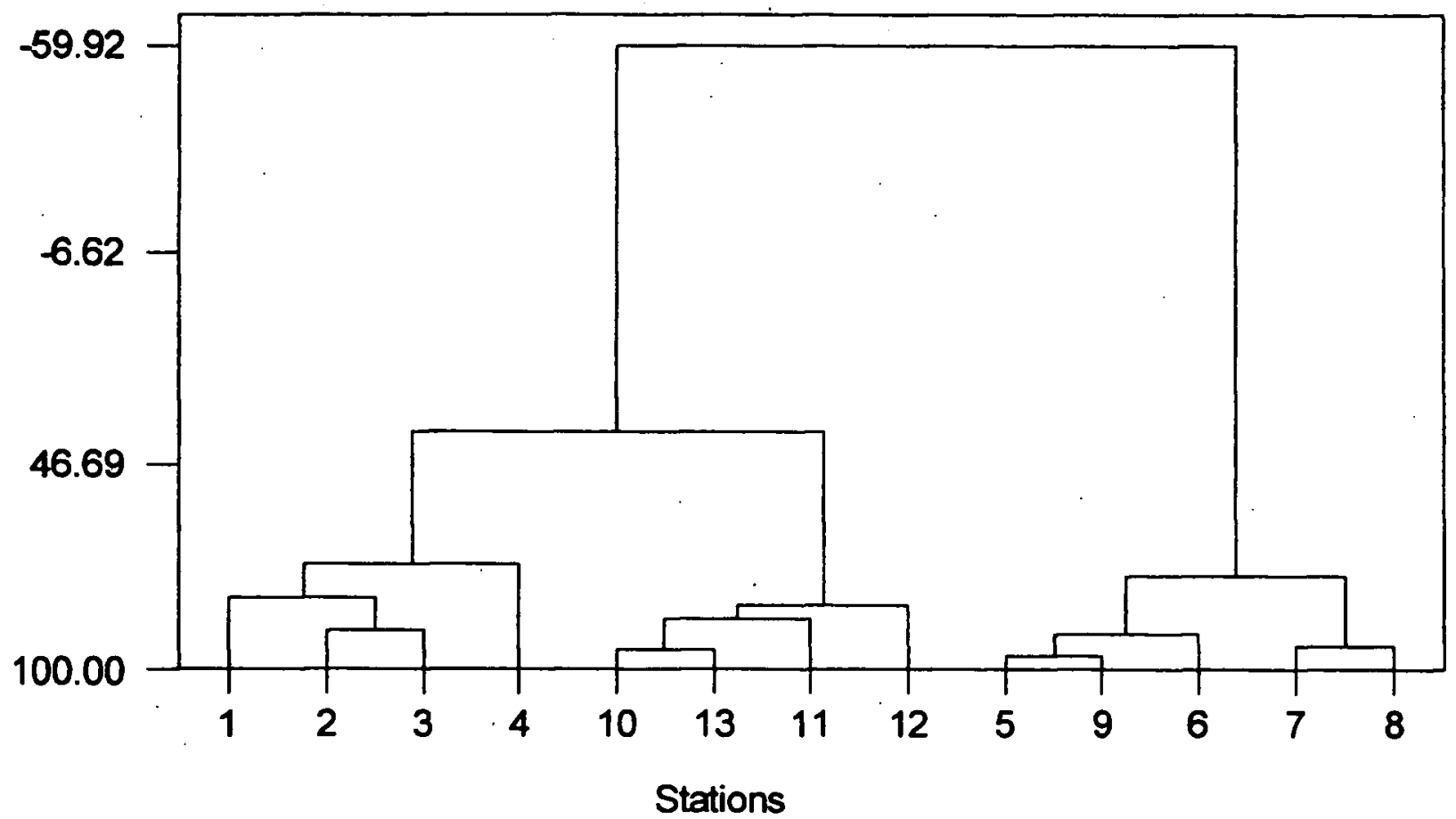

Fig. 3. Dendrogramme des stations classées en fonction des 12 principaux paramètres physico-chimiques.

Fig. 3. Dendrogram of sampling sites clustered for 12 physico-chemical variables. 
té : $109 \mu \mathrm{S} / \mathrm{cm})$ et proches de la neutralité $(\mathrm{pH}: 7,3)$. Les teneurs en orthophosphates y sont relativement faibles ([P-PO43-] : $130 \mu \mathrm{g} / \mathrm{l})$;

- le groupe 2 (M10, M11, M12, M13) contient 4 sites méso-eutrophes $\left(\left[\mathrm{N}-\mathrm{NH}_{4}{ }^{+}\right]: 120 \mu \mathrm{g} / 1 ;\left[\mathrm{P}_{-} \mathrm{PO}_{4}{ }^{3-}\right]: 130\right.$ $\mu \mathrm{g} / \mathrm{l})$ situés sur la Zinsel du Nord en plaine d'Alsace ;

- le groupe 3 (M5, M6, M7, M8, M9) comprend les 4 sites localisés sur la Moder en plaine d'Alsace et la station du Rothbach (M9). Il regroupe des stations aux eaux minéralisées (conductivité : $250 \mu \mathrm{S} / \mathrm{cm}$ ), alcalines $(\mathrm{pH}: 7,8)$ et enrichies en orthophosphates ([P$\left.\left.\mathrm{PO}_{4}{ }^{3-}\right]: 275 \mu \mathrm{g} / \mathrm{l}\right)$.

Les sites se distribuent de l'amont vers l'aval selon un gradient croissant de minéralisation et secondairement de trophie.

\subsection{Composition floristique de la Moder}

L'A.F.C. établie sur une matrice «espèces $\mathrm{x}$ stations» a mis en évidence une distribution des végétaux selon un axe $1(\mathrm{~F} 1=33,3 \%)$ correspondant à une zonation de l'amont (Vosges du Nord) vers l'aval (plaine d'Alsace) de la Moder (Fig. 4). A droite, les bryophytes telles Amblystegium fluviatile et Chiloscyphus polyanthos colonisent les têtes de bassin versant alors qu'à gauchè des algues du genre Cladophora et des phanérogames telles Myriophyllum spicatum L. ou Elodea ernstae St-John caractérisent l'aval. L'axe 2 (F2 = $22,5 \%$ ) oppose des amphiphytes comme Berula erecta (Hudson) Coville, ou Glyceria fluitans L. présents à la source de la Moder (M1, M2) aux hydrophytes phanérogames telles Potamogeton crispus L. ou Ranunculus peltatus Schrank caractérisant les affluents de la Moder en plaine d'Alsace (Fig. 4). L'axe 2 oppose ainsi la Moder à ses affluents en plaine (stations M10 à M13).

3.2.1. Composition floristique des stations des Vosges $d u$ Nord (Tableau 2)

Des hélophytes de bordure, Chrysosplenium oppositifolium L., C. alterniflorum L., Stellaria alsine Grimm caractérisent la source de la Moder. Cette tête de bassin localisée en milieu forestier ne favorise que peu l'installation des bryophytes en raison de l'ensablement important du lit. Quelques blocs sont colonisés par Fissidens crassipes Loeske, Rhynchostegium riparioides Hedw., Amblystegium riparium Hedw. et A. tenax Hedw. (station M1). Des amphiphytes Berula erecta, Glyceria fluitans et des callitriches (C. stagnalis Scop., C. platycarpa Kütz et $C$. obtusangula Le Gall), Sparganium emersum Rehmann colonisent ce ruisseau peu profond (station M1). A l'aval (M2) Nasturtium officinale $\mathrm{R}$. Br. forme des cressonnières denses dans les zones bien éclairées où il est alors ac- compagné par Carex rostrata Stokes, Scirpus sylvaticus L. et Juncus effusus L. A l'aval de ce secteur (M3, M4), le cortège floristique est constitué par des amphiphytes (Berula erecta, Glyceria fluitans), par des hydrophytes phanérogames (Ranunculus peltatus, Elodea canadensis Michaux, E. nuttallii St-John, Callitriche stagnalis, C. platycarpa, C. obtusangula, C. hamulata Koch et Sparganium emersum) et localement par des bryophytes (Amblystegium riparium., A. fluviatile, A.tenax, Rhynchostegium riparioides, Chiloscyphus polyânthos L., Fontinalis antipyretica Hedw.).

3.2.2. Composition floristique des affluents de la Moder en plaine d'Alsace

Malgré une minérălisation plus élevée, les affluents de la Moder renferment sensiblement les mêmes espèces d'hydrophytes phanérogames que dans les Vosges du Nord (Tableau 2). En effet, Ranunculus peltatus, Callitriche hamulata, C. platycarpa, C. obtusangula, Elodea canadensis (présente localement), E. nuttallii et Vaucheria sp. sont les principales espèces rencontrées sur ces affluents. Le cortège bryophytique est généralement constitué de Fontinalis antipyretica, Rhynchostegium riparioides, Amblystegium riparium et A. tenax. Lorsque les conditions morphométriques et la physico-chimie le permettent, Potamogeton crispus s'y ajoute (stations M9 à M13). La présence de Fissidens crassipes et surtout d'algues filamenteuses ( $\mathrm{Cla}$ dophora sp., Vaucheria sp.) rapproche le Rothbach (station M9) des stations de la Moder en plaine d'Alsace (Fig. 4). En revanche, la composition floristique de la Zinsel du Nord (stations M10 à M13) se distingue nettement de la Moder dans les Vosges du Nord et en plaine d'Alsace (Fig. 4).

\subsubsection{Composition floristique de la Moder en plaine d'Alsace}

En plaine d'Alsace, les stations se caractérisent par les espèces de macrophytes aquatiques déjà présentes dans les tronçons aval de la Moder gréseuse (Tableau 2). A ce groupement végétal s'ajoute la présence d'un autre genre d'algue Cladophora sp., et d'une autre espèce d'élodée, Elodea ernstae. En revanche, Ranunculus peltatus n'est plus observé sur la Moder en plaine d'Alsace (stations M5 à M8). Amblystegium fluviatile et Chiloscyphus polyanthos sont également absents des stations aval.

\subsection{Relations entre les caractéristiques physico- chimiques et la composition floristique}

Les relations entre les espèces floristiques et les variables physico-chimiques ont été testées d'une part entre le premier axe de l'ACP et d'autre part le premier axe de l'AFC et entre le second axe de l'ACP et le se- 


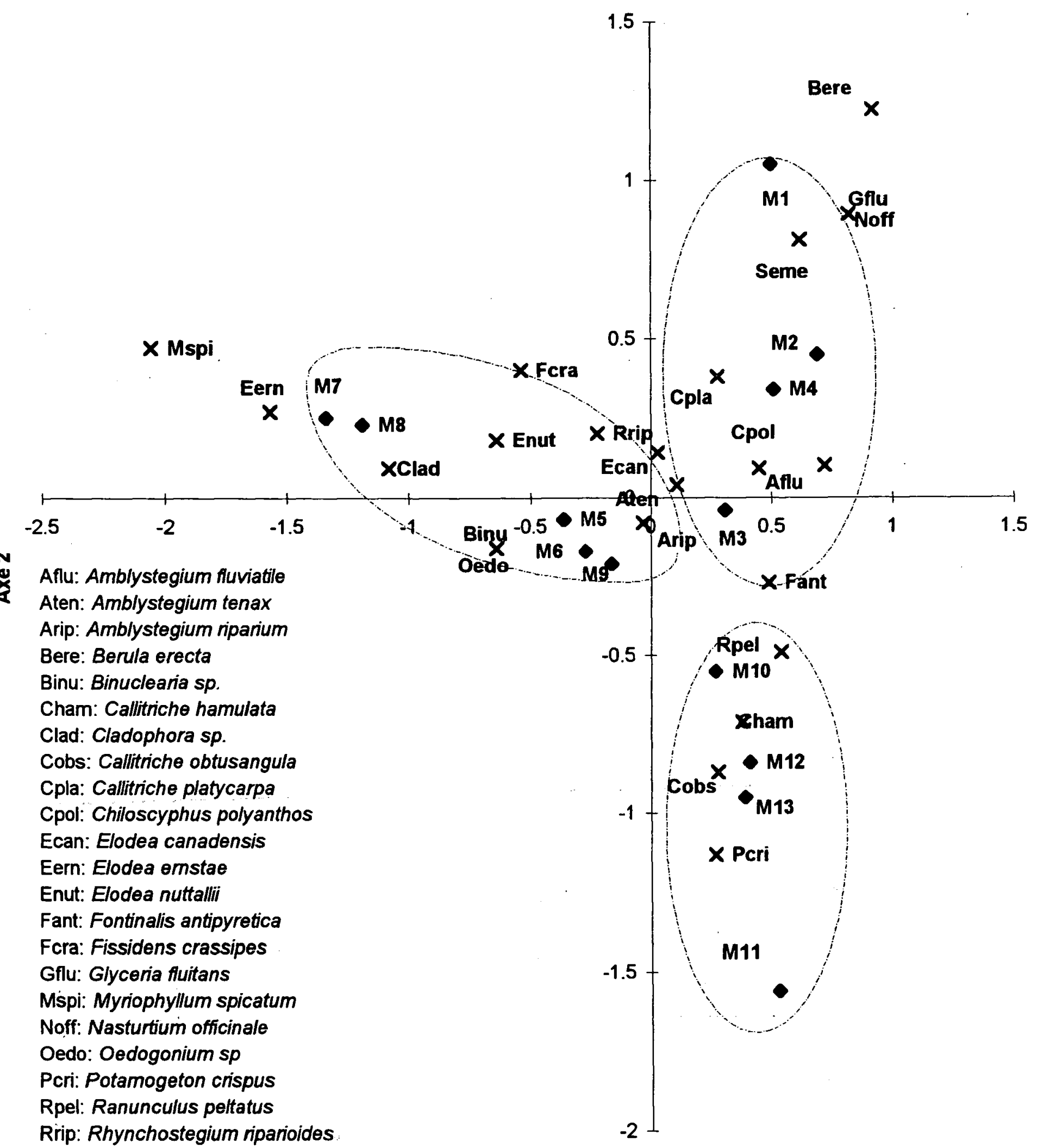

Seme: Sparganium emersum

Axe 1

Fig. 4. Analyse Factorielle des Correspondances AFC sur les hydrophytes. Position des 13 relevés et 23 espèces floristiques. Fig. 4. Ordination of samples plots using FCA of floristic data. 
Tableau 2. Relations entre la composition physico-chimique de la Moder et les macrophytes aquatiques.

Parenthèses : espèces avec une faible fréquence.

Les écarts-types des principaux paramètres physico-chimiques sont calculés à partir de l'ensemble des valeurs et non pas sur les valeurs moyennes.

Table 2. Relationships between aquatic macrophyte communities and physico-chemical composition of the river Moder. Bracket : species with a low frequency.

Standard deviations were calculated on all physico-chemical data.

\begin{tabular}{|c|c|c|c|c|c|}
\hline & \multicolumn{3}{|c|}{ YOSELS BU WOMB: } & \multicolumn{2}{|c|}{ 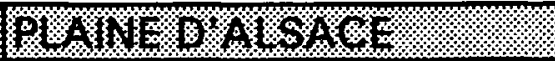 } \\
\hline & 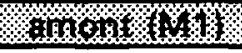 & 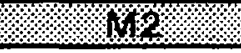 & W wa & 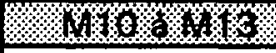 & , 1456 \\
\hline N. officinale & + & + & + & & \\
\hline C. stagnalis & + & + & + & & \\
\hline C.hamulata & & + & + & + & $1+1$ \\
\hline C. platycarpa & + & + & + & + & $1+1$ \\
\hline F. crassipes & + & & + & & + \\
\hline A. riparium & + & + & + & + & + \\
\hline A. tenax & + & + & + & + & + \\
\hline R. riparioides & + & + & + & $(+)$ & $(+1$ \\
\hline C. hamulata & $1+1$ & + & + & + & $(+)$ \\
\hline C. platycarpa & + & + & + & + & $(+)$ \\
\hline A, fluviatile & & + & + & $(+)$ & \\
\hline C. polyanthos & & + & $1+1$ & $(+)$ & \\
\hline E. canadensis & & + & $1+1$ & $(+)$ & \\
\hline E. nuttallii & & $1+1$ & + & $(+)$ & $(+)$ \\
\hline F. antipyretica & & + & + & + & $(+)$ \\
\hline R. peltatus & & + & + & + & \\
\hline C. obtusangula & & & + & + & + \\
\hline Vaucheria sp. & & & + & + & + \\
\hline Cladophora sp. & & & & & + \\
\hline P. crispus & & & & + & \\
\hline E.ernstae & & & & & + \\
\hline $\mathrm{pH}$ & $7,2+1 \cdot 0,1$ & $7,3+1-0,2$ & $7.3+1-0.2$ & $7,6+1-0,3$ & $7,8+1-0,2$ \\
\hline conductivité $\mu \mathrm{S} / \mathrm{cm}$ & $95+1-7,5$ & $130+1-32$ & $108+/-6$ & $18+1-34$ & $252+1-48$ \\
\hline$\left[\mathrm{N}-\mathrm{NH}_{4}{ }^{*}\right] \mu \mathrm{g} / \mathrm{l}$ & $76+1-59$ & $154+1-69$ & $268+\%-244$ & $122+/-105$ & $205+/-102$ \\
\hline$\left[\mathrm{P}_{-} \mathrm{PO}_{4}^{3}-\right] \mu \mathrm{g} / \mathrm{l}$ & $90+1-33$ & $85+1-16$ & $166+1-78$ & $134+1.49$ & $275+1.78$ \\
\hline$\left[\mathrm{N}-\mathrm{NO}_{3}\right] \mathrm{mg} / \mathrm{l}$ & $0,9+1-0,1$ & $1,1+1-0,2$ & $1,0+1-0,2$ & $0,8+1-0,2$ & $1,8+1-0,7$ \\
\hline Géologie & & Grès & & Marnes & \\
\hline
\end{tabular}

cond axe de l'AFC (Fig. 5). Elles sont significatives (test de Fisher, rejet de l'hypothèse $\mathrm{a}=0$ au seuil de $1 \%$ pour l'axe 1 et au seuil $6 \%$ pour l'axe 2 ). Il existe donc des relations significatives entre la distribution des espèces et la composition physico-chimique des stations (Fig. 5). Les trois groupes de stations individualisés par la CAH constituent des ensembles floristiques homogènes, représentés sur la figure 4 :

- un groupe constitué par la Moder dans les Vosges du Nord (M1 à M4) ;
- un groupe formé par la Zinsel du Nord (M10 à M13);

- un groupe renfermant les stations de la Moder en plaine d'Alsace (M5 à M8) et son affluent le Rothbach (M9). La composition physico-chimique (teneurs élevées en orthophosphates) et floristique (présence d'algues filamenteuses) de la station M9 explique son classement avec les stations de la Moder en plaine d'Alsace. 

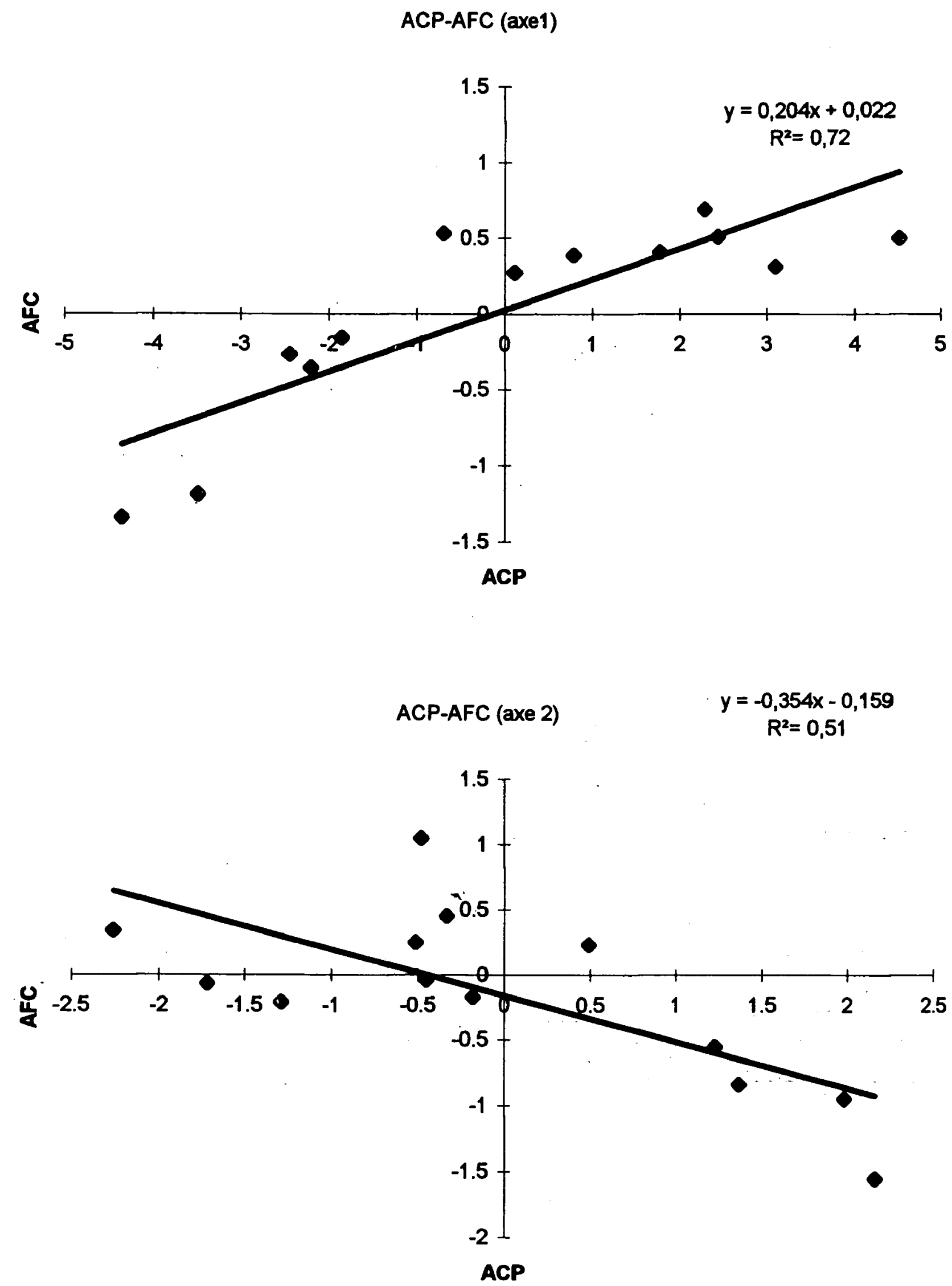

Fig. 5. Relations entre les axes de l'ACP et de l'AFC.

Fig. 5. Relationships between PCA and FCA axes. 


\section{Discussion}

\subsection{Caractéristiques physico-chimiques et floris- tiques de la Moder}

La Moder ne possède plus les mêmes caractéristiques morphométriques et physico-chimiques en plaine que dans les Vosges du Nord. En effet, les sites aval des Vosges du Nord sont proches de la neutralité (M1M4 pH : 7,3), alors qu'ils sont davantage alcalins en plaine (M5-M8 pH : 7,8). L'enrichissement en minéraux à l'aval de la Moder s'explique essentiellement par les teneurs en calcium sensiblement plus élevées (Vosges du Nord $\left(\mathrm{Ca}^{2+}\right)<10 \mathrm{mg} / 1$; Moder aval $\left(\mathrm{Ca} 2^{+}\right)$ $=40 \mathrm{mg} / \mathrm{l})$. La différence de nature du substratum géologique entre la Moder amont et aval explique la minéralisation différente entre les deux secteurs géologiques (Vosges du Nord : conductivité $110 \mu \mathrm{S} / \mathrm{cm}$; Moder aval : $260 \mu \mathrm{S} / \mathrm{cm}$ ). La nature siliceuse de la roche-mère des Vosges du Nord donne naissance à des eaux pauvres en bases, neutres et peu tamponnées. En revanche; les marnes et marno-calcaires du substratum géologique de la plaine conduisent à un enrichissement en minéraux (particulièrement en calcium) et à une alcalinisation des eaux.

La Moder reçoit dès l'amont (M1) des effluents domestiques chargés en nutriments, qui expliquent les fortes concentrations en azote ammoniacal et en orthophosphates de ce tronçon (M1 : $\left(\mathrm{N}-\mathrm{NH}_{4}{ }^{+}\right): 80 \mu \mathrm{g} / \mathrm{l}$; $\left.\left(\mathrm{P}-\mathrm{PO}_{4}{ }^{3-}\right): 90 \mu \mathrm{g} / \mathrm{l}\right)$. A l'aval des bassins de piscicultures (M2) et des villages (M3, M4), les concentrations en nutriments augmentent et atteignent des valeurs élevées $\left(\mathrm{M} 4:\left(\mathrm{N}_{-} \mathrm{NH}_{4}{ }^{+}\right): 440 \mu \mathrm{g} / \mathrm{l} ;\left(\mathrm{P}_{-} \mathrm{PO}_{4}{ }^{3-}\right): 210 \mu \mathrm{g} / \mathrm{l}\right)$. Cet enrichissement en nutriments est d'origine anthropique. L'augmentation dès teneurs en phösphorè résulte des activités humaines (lessives essentiellement). Le rôle du phosphore dans l'eutrophisation des cours d'eau a par ailleurs été clairement démontré (Carbiener 1990, Caraco 1993). De même, Trémolières et al. (1994) et Daniel \& Haury (1996) ont montré l'enrichissement en azote ammoniacal résultant des activités piscicoles. En plaine d'Alsace (stations M5 à M13), de nouvelles sources de pollution apparaissent (activités agricoles et industrielles, urbanisation) et expliquent que les concentrations en nutriments demeurent élevées. Le niveau trophique n'est pas sensiblement différent en plaine de celui mesuré dans les zones aval des Vosges du Nord.

La végétation aquatique reflète la qualité de l'eau et plus particulièrement, le niveau trophique (Carbiener et al. 1990, Muller 1990, Robach et al. 1996, Thiébaut 1997). En effet, le niveau trophique élevé du cours d'eau dans les Vosges du Nord est confirmé par les herbiers très importants à Ranunculus peltatus (espèce dont l'optimum de développement est atteint dans les eaux eutrophes des Vosges du Nord) et à Callitriche obtusangula (espèce réputée eutrophe), alliés à la présence d'algues du genre Vaucheria, de bryophytes telles Amblystegium riparium, Fissidens crassipes ainsi que le remplacement quasi total vers l'aval d'Elodea canadensis par $E$. nuttallii, espèce réputée ammoniophile (Dendène et al. 1993). Les sites aval situés sur grès se caractérisent par une biomasse importante alors que ceux situés en plaine d'Alsace ne sont que peu végétalisés. Potamogeton crispus n'est présent que ponctuellement dans quelques ruisseaux des Vosges du Nord et sur leurs affluents en plaine. Grasmuck (1994) a montré que cette espèce peut supporter une forte minéralisation (conductivité $>1000 \mu \mathrm{S} / \mathrm{cm}$ ) et des teneurs

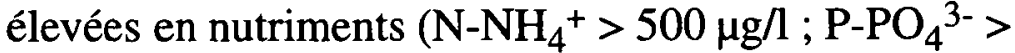
$500 \mu \mathrm{g} / \mathrm{l})$ dans les cours d'eau lorrains. Ce potamot possède donc une large amplitude écologique vis-à-vis de la minéralisation et de la trophie. De même, Ranunculus peltatus, absent sur la Moder en plaine, colonise la Moder amont et ses affluents en plaine. Grasmuck (1994) a établi que Ranunculus peltatus disparait des eaux dont la conductivité dépasse $600 \mu \mathrm{S} / \mathrm{cm}$ et devient plus fréquent et abondant lorsque les conductivités sont inférieures à $100 \mu \mathrm{S} / \mathrm{cm}$. L'absence de Ranunculus peltatus en plaine d'Alsace et dans les rivières phréatiques pourrait s'expliquer par les teneurs trop élevées en calcium des eaux.

L'absence de Potamogeton crispus sur la Moder en plaine d'Alsace pourrait alors être attribuée à la morphométrie du lit de la rivière (absence d'habitat suite au recalibrage) alors que la non présence de Ranuncu-lus peltatus pourrait s'expliquer par la nature de la roche-mère et la minéralisation élevée. Des expériences de transplantations de ces deux espèces permettraient de confirmer ou non ces deux hypothèses. Des études en milieu contrôlé de laboratoire devraient permettre de préciser le rôle de la minéralisation sur le développement de Ranunculus peltatus.

\subsection{Comparaison avec les rivières phréatiques du Ried alsacien}

\subsubsection{Comparaison de la composition physico-chi- mique des deux secteurs}

Les rivières phréatiques du Ried alsacien sont situées en plaine alluviale ello-rhénane (Nord-Est de la France). Le réseau hydrographique draine un substrat bicarbonatế calcique présentant une relative homogénéité vis-à-vis de la minéralisation $(\mathrm{pH} 7,5$ à 8,0 ; conductivité : $500-700 \mu \mathrm{S} / \mathrm{cm}$ ). Les cours d'eau, circulant essentiellement en milieu prairial, correspondent à des eaux de surface et de rivières phréatiques, 
principalement alimentées par des résurgences de la nappe phréatique des graviers glaciaires du Rhin. Les sources issues directement de la nappe possèdent des caractéristiques physico-chimiques relativement constantes. Les eaux limpides; pures, fraîches (9-13 ${ }^{\circ} \mathrm{C}$ ), oligosaprobes et oligotrophes aux sources, subissent un enrichissement en nutriments suite aux activités piscicoles, agricoles, industrielles et à l'urbanisation du secteur (Carbiener et al. 1990, Trémolières et al. 1993, 1994).

Dans le Ried alsacien, les cours d'eau sont donc davantage minéralisés (conductivité : $500-700 \mu \mathrm{S} / \mathrm{cm}$ ) que la Moder et ses affluents en plaine (entre 100 et $300 \mu \mathrm{S} / \mathrm{cm}$ ). En effet, les teneurs en calcium et en magnésium sont nettement plus faibles dans le réseau hydrographique de la Moder aval $\left(\mathrm{Ca}^{2+}=20\right.$ à $50 \mathrm{mg} / \mathrm{l}$; $\mathrm{Mg}^{2+}=4$ à $\left.10 \mathrm{mg} / \mathrm{l}\right)$ que dans le Ried alsacien $\left(\mathrm{Ca}^{2+}=\right.$ 80 à $100 \mathrm{mg} / \mathrm{l} ; \mathrm{Mg}^{2+}=15$ à $\left.20 \mathrm{mg} / \mathrm{l}\right)$. La nature différente du substrat géologique (marnes en plaine et galets bicarbonatés calciques dans le Ried) explique cette différence de minéralisation. Le niveau trophique des zones aval (types D, E et F, Robach et al. 1996) des rivières phréatiques $\left(\mathrm{N}-\mathrm{NH}_{4}{ }^{+}=35\right.$ à $255 \mu \mathrm{g} / 1 ; \mathrm{P}$ $\mathrm{PO}_{4} 3^{-}=30$ à $190 \mu \mathrm{g} / \mathrm{l}$ ) est par contre semblable aux valeurs mesurées sur le bassin versant de la Moder (N$\mathrm{NH}_{4}{ }^{+}=55$ à $440 \mu \mathrm{g} / 1 ; \mathrm{P}_{-} \mathrm{PO}_{4} 3-=80$ à $\left.300 \mu \mathrm{g} / \mathrm{l}\right)$. De même, ces secteurs eutrophes du Ried renferment des concentrations en azote nitrique semblables à celles mesurées dans la Moder aval $\left(\mathrm{N}-\mathrm{NO}_{3}{ }^{-}=0,8\right.$ à 2,2 $\mathrm{mg} / \mathrm{l})$. Le Ried et, dans une moindre mesure, la Moder aval sont des secteurs agricoles : l'azote nitrique mesuré peut être dû à des apports d'engrais sous forme de nitrates (Carbiener et al. 1990, Trémolières et al. 1994). Il apparaît donc que c'est la nature du substrat géologique qui différencie particulièrement la Moder et les rivières phréatiques du Ried alsacien.

\subsubsection{Comparaison de la composition floristique des} deux secteurs

Les espèces Elodea canadensis, E. nuttallii, Callitriche obtusangula, Fontinalis antipyretica, Amblystegium riparium et Vaucheria sp. sont communes à la Moder et aux rivières du Ried alsacien (Tableau 3). Par contre, Elodea ernstae et Cladophora sp. sont présents uniquement dans les eaux moyennement minéralisées et eutrophes de la Moder à fortement minéralisées et hypertrophes du Ried alsacien. Callitriche obtusangula est considéré comme espèce eutrophe dans les cours d'eau faiblement minéralisés des Vosges du Nord (Muller 1990, Thiébaut \& Muller 1996) alors qu'il apparaît dans les rivières phréatiques pour des concentrations en nutriments très faibles $\left(\mathrm{N}-\mathrm{NH}_{4}{ }^{+}>11 \mu \mathrm{g} / \mathrm{l} ; \mathrm{P}-\right.$ $\mathrm{PO}_{4}{ }^{3-}>7 \mu \mathrm{g} / \mathrm{l}$; Carbiener et al. 1990, Trémolières et al. 1993, Robach et al. 1996). Duvigneaud \& Schotsman (1977) ont constaté que, dans les ruisseaux ou petites rivières enrichies en matières organiques, $C$. obtusangula ne fleurit pas et manifeste une vitalité amoindrie. Ils n'ont pas pu établir si cette réaction était liée au phénomène même de pollution organique ou à l'élévation de température qui l'accompagne généralement. Une différence d'amplitude écologique vis-à-vis de la trophie entre milieu faiblement et fortement minéralisé se retrouve également pour d'autres espèces (Robach et al. 1996). En effet, Amblystegium riparium se développe pour des teneurs élevées en nutriments dans les eaux peu minéralisées (Vosges du Nord : Thiébaut 1997, Bretagne : Haury \& Muller 1991) alors que cette mousse apparaît dans des eaux mésotrophes en milieu fortement minéralisé du Ried alsacien (Carbiener et al. 1990). L'absorption du phosphore serait favorisée dans les eaux alcalines par la richesse en calcium, les plantes auraient donc besoin d'utiliser des concentrations supérieures en phosphore dans les eaux pauvres en calcium. Les interactions entre la minéralisation et la trophie pour certains macrophytes aquatiques comme Callitriche obtusangula pourraient être également étudiées en conditions contrôlées de laboratoire.

Les rivières phréatiques possèdent toutefois une plus grande diversité floristique (Robach et al. 1996). Ainsi Ranunculus fluitans Lam., Potamogeton perfoliatus L., P. pectinatus L., P. lucens L., P. friesii Rupr., $P$. nodosus Poiret, Ceratophyllum demersum L. sont des espèces caractéristiques des rivières phréatiques bicarbonatées calciques et eutrophes (type F), qui manquent totalement dans la Moder aval, bien que le niveau trophique soit semblable entre ces deux réseaux hydrographiques. L'absence dans la Moder de végétaux inféodés aux eaux fortement minéralisées du Ried alsacien tels Ranunculus fluitans, Potamogeton perfoliatus, $P$. pectinatus, 'P. lucens pourrait s'expliquer par les trop faibles teneurs en cations (calcium notamment) dans cette rivière. Par contre, la Moder abrite des espèces liées aux eaux faiblement acides, comme Callitriche hamulata, C. platycarpa, C. stagnalis, Ranunculus peltatus, qui manquent totalement dans les eaux bicarbonatées calciques des cours d'eau du Ried.

\section{Conclusion}

La Moder subit dès l'amont des perturbations liées à l'anthropisation du milieu déterminant un niveau trophique élevé. De l'amont vers l'aval, les groupements végétaux se répartissent selon un gradient croissant de minéralisation. La composition floristique reflète le niveau trophique élevé dès l'amont. Une comparaison entre la.composition floristique de la Moder et celles 
Tableau 3. Comparaison entre les phytocénoses aquatiques de la Moder (Vosges du Nord, Plaine d'Alsace) et celles des rivières phréatiques du Ried alsacien.

Parenthèses : espèces avec une faible fréquence Les espèces communes entre la Moder et le Ried alsacien sont représentées en gras. Les données floristiques et physico-chimiques du Ried alsacien sont extraites de Robach et al. (1996).

Table 3. Comparison between aquatic macrophyte communities of the river Moder and Ried alsatian rivers.

Bracket : species with a low frequency. Species common to the Moder and Ried rivers are written in bold characters. The floristic and physico-chemical data of the Ried river are taken from Robach et al. (1996).

\begin{tabular}{|c|c|c|c|c|c|c|}
\hline & \multicolumn{3}{|c|}{ MEN } & \multicolumn{3}{|c|}{ 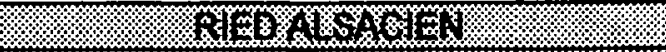 } \\
\hline & \multirow{2}{*}{ 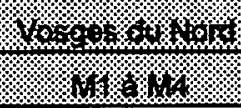 } & \multicolumn{2}{|c|}{ 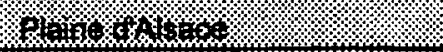 } & \multicolumn{3}{|c|}{ S S } \\
\hline & & 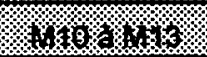 & 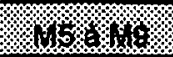 & 8. & সax: & max: \\
\hline \multicolumn{7}{|c|}{ Espèces présentes seulement sur la Moder } \\
\hline C. stagnalis & + & & & & & \\
\hline A. fluviatile & $(+)$ & $(+)$ & & & & \\
\hline C. polyanthos & $(+)$ & $(+)$ & & & & \\
\hline R. peltatus & + & + & & & & \\
\hline A. tonax & + & + & + & & & \\
\hline F. crassipes & $(+)$ & & + & & & \\
\hline R. riparioides & + & $(+)$ & $(+)$ & & & \\
\hline C. hamulata & $(+)$ & + & $(+)$ & & & \\
\hline C. platycarpa & + & + & $(+)$ & & & \\
\hline \multicolumn{7}{|c|}{ Espèces communes aux deux secteurs } \\
\hline C. obtusangula & + & + & + & + & + & + \\
\hline E. canadensis & $(+)$ & $(+)$ & $(+)$ & + & + & $(+)$ \\
\hline E. nuttallii & + & $(+)$ & $(+)$ & + & + & + \\
\hline A. niparium & + & + & + & $(+)$ & $(+)$ & $(+)$ \\
\hline F. antipyretica & + & + & $(+)$ & + & + & \\
\hline Vaucheria sp. & + & & + & + & + & + \\
\hline P. crispus & & $(+)$ & & + & + & + \\
\hline E.emstae & & & $(+)$ & & + & $(+)$ \\
\hline M. spicatum & & & $(+)$ & + & + & + \\
\hline Cladophora sp. & & & + & + & + & + \\
\hline \multicolumn{7}{|c|}{ Eșpèces présentes uniquement dans le Ried alsacien } \\
\hline L. trisulca & & & & + & + & \\
\hline P.friesii & & & & + & + & + \\
\hline A.filliculoides & & & & + & + & + \\
\hline S. polymiza & & & & + & + & + \\
\hline C. demersum & & & & + & + & + \\
\hline P. pectinatus & & & & + & + & + \\
\hline G. densa & & & & + & + & \\
\hline M. verticillatum & & & & + & + & \\
\hline P. perfoliatus & & & & + & + & + \\
\hline R. fuitans & & & & & + & + \\
\hline P. lucens & & & & & & + \\
\hline P.nodosus & & & & & & + \\
\hline $\mathrm{pH}$ & $7,3+/-0,2$ & $7.6+1-0.3$ & $7,8+1-0,2$ & $7,6+1-0,2$ & $7,9+1-0,2$ & $7,9+1-0,2$ \\
\hline conductivité $\mu \mathrm{S} / \mathrm{cm}$ & $110+1-22$ & $18+1-34$ & $252+1-48$ & $657+1-66$ & $657+1-63$ & $508+1-52$ \\
\hline$\left[N-N_{4}^{*}\right] \mu g /$ & $210+/-215$ & $122+/-105$ & $205+1-102$ & $34+1-31$ & $61+1-40$ & $255+/-107$ \\
\hline 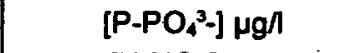 & $130+1-71$ & $134+/-49$ & $275+1-78$ & $29+1-24$ & $40+1-33$ & $192+/-116$ \\
\hline$\left[\mathrm{N}-\mathrm{NO}_{3}\right] \boldsymbol{\mu g} /$ & $1,0+1-0,2$ & $0,8+1-0,2$ & $1,8+1-0,7$ & $2,9+1-2,5$ & $1,6+l-1,1$ & $2,5+1-0,9$ \\
\hline Géologie & Grès & Mames & & & carbonatés c & \\
\hline
\end{tabular}


entre la composition floristique de la Moder et celles des rivières phréatiques du Ried alsacien met en évidence la présence d'espèces communes (Callitriche obtusangula, Elodea canadensis, E. nuttallii, Amblystegium riparium, Fontinalis antipyretica, et Vaucheria sp.) déterminées par des niveaux trophiques similaires et des différences liées aux variations de minéralisation (présence de Ranunculus peltatus, Callitriche hamulata, $C$. platycarpa, C. stagnalis dans la Moder; de Ranunculus fluitans, Potamogeton nodosus, $P$. pectinatus, $P$. lucens, etc, dans le Ried alsacien). Cette étude confirme donc le rôle essentiel de la minéralisation dans la composition floristique des phytocénoses aquatiques des systèmes eutrophisés.

\section{Travaux cités}

AFNOR 1990. - Recueil de normes françaises. Eaux-Méthodes d'essais. $4^{\text {ème }}$ éd. Paris : 736p.

Barroin G. 1991. — La réhabilitation des plans d'eau. La Recherche, 238 (22) : 1412-1422.

Bornette G. \& Amoros C., 1991. - Aquatic vegetation and hydrology of a braided river floodplain. J. Veg. Sci., 2, 497-512.

Bourrelly P., 1968, 1970, 1990. - Les algues jaunes et brunes, Les algues bleues et rouges,. Les algues vertes. Boubée, Paris, pages multiples.

Braun-Blanquet J. 1964. - Pflanzensoziologie. Springer Verlag, Wien : $865 \mathrm{p}$.

Caraco N. F. 1993. - Disturbance of the phosphorus cycle : A case of indirect effects of human activity. Tree, 8, 2, $4 \mathrm{p}$.

Carbiener R. 1990. - Compositions lessivielles avec ou sans phosphates et protection des milieux aquatiques. Rapport au Secrétariat d'Etat du Ministère de l'Environnement, $182 \mathrm{p}$.

Carbiener R., Trémolières M. \& Muller S. 1996. - Végétation des eaux courantes et qualité des eaux : une thèse, des débats, des perspectives. Acta bot. Gallica, 142 (6), 489-531.

Carbiener R., Trémolières M., Mercier J.-L. \& Ortscheit A. 1990. Aquatic macrophyte communities as bioindicators of eutrophication in calcareous oligosaprobe stream waters (Upper Rhine plain, Alsace). Vegetatio, 86, 71-88.

Daniel H. \& Haury J. 1996. - Effects of fish farm pollution on phytocenoses in an acidic river (the River Scorff, South Brittany, France). Acta bot. Gallica, 142 (6), 639-650.

Dendène M.-A., Rolland T., Trémolières M. \& Carbiener R. 1993. - Effect of ammonium ions on the net photosynthesis of three species of Elodea. Aquat. Bot., 46, 301-315.

Duvigneaud J. \& Schotsman H. D. 1977. - Le genre Callitriche en Belgique et dans les régions avoisinantes. Nouvelles contributions et clé de détermination. Natura Mosana, 30 (1), 1-21.

Grasmuck N. 1994. - La végétation aquatique des cours d'eau de Lorraine : typologie floristique et écologique. Contribution a l'étude de l'autoécologie des espèces de la flore aquatique lorraine. Thèse Doctorat : Univ. de Metz : 248 p. + annexes.

Harding J.P.C 1981. - Macrophytes as monitors of river quality in the Southern N.W.W.A area, North West Water Authority, Rivers Divisions, Réf $\mathrm{n}^{\circ}$ TS-BS-81-2, 1-54.

Haslam S. H. 1987. - River Plants of Western Europe. Cambridge University Press : 512p.

Haslam S. M. \& Wolseley P. A. 1981. - River vegetation, its identification. Assessment and Management. Cambridge University Press, Cambridge : 1-15.

Haury J. 1985. — Etude écologique des macrophytes du Scorff (Bretagne Sud). Thèse Doctorat Rennes I : 240p.
Haury J. \& Muller S. 1991. - Variations écologiques et chorologiques de la végétation macrophytique des rivières acides du Massif armoricain et des Vosges du Nord (France). Rev. Sci. Eau, 4, 463-482.

Haury J., Peltre M.-C., Muller S., Trémolières M., Barbe J., Dutartre A. \& Guerlesquin M. 1996. - Des indices macrophytiques pour estimer la qualité des cours d'eau : premières propositions. Ecologie, 27 (4), 233-244.

Hutchinson G.E. 1975. - A Treatise on Limnology. Limnological Botany, New Haven, $3: 660$ p.

Kohler A., Zeltner G. \& Wonneberger R. 1973. - Die Bedeutung chemischer und pflanzlicher Verschmutzungsindikatoren im Fließgewässersystem Moosbach (Münchener Ebene). Arch. Hydrobiol., 72, 533-549.

Lambinon J., De Langhe J. E, Delvosalle L., Duvigneaud J. \& Vanden Berghen C. 1992. - Nouvelle Flore de la Belgique, du GrandDuché de Luxembourg, du Nord de la France et des Régions Voisines (Ptéridophytes et Spermaphytes). Editions du Patrimoine du Jardin botanique national de Belgique, Meise, 4 ème édit. : 1092 p.

Lévèque C. 1994. - Etat de santé des écosystèmes aquatiques : l'intérêt des variables biologiques. In : Séminaire national «Les variables biologiques : des indicateurs de l'état de santé des écosystèmes aquatiques», 2-3 nov. 1994, Paris : 12-25.

Moss B., Johnes P. \& Phillips G. 1996. - The monitoring of ecological quality and the classification of standing waters in temperate regions : a review and proposal based on a worked scheme for British waters. Biol. Rev., 71, 301-339.

Muller S. 1990. - Une séquence de groupements végétaux bioindicateurs d'eutrophisation croissante des cours d'eau faiblement minéralisés des Basses Vosges gréseuses du Nord. C. R. Acad. Sci. Paris, 310, Série III : 509-514.

Newbold C. \& Holmes N. T. H., 1987. - Nature conservation : water quality criteria and plants as water quality monitors. Water Pollution Control, 86 (2) : 345-364.

Peltre M.-C. \& Léglize L. 1992. - Essais d'application d'un protocole hiérarchisé pour l'étude des peuplements végétaux aquatiques en eau courante. Annales Nationales de la Protection des Plantes, XV'me conférence du COLUMA, Versailles : 1049-1057.

Robach F., Thiébaut G., Trémolières M. \& Muller S. 1996. - A reference system for continental running waters : plant communities as bioindicators of increasing eutrophication in alkaline and acidic waters in North East of France. Hydrobiologia, 340 : 67-76.

Smith A.J. E. 1992. - The liverworts flora of Britain and Ireland. Cambridge University Press., Cambridge : $362 \mathrm{p}$.

Thiébaut G. \& Muller S. 1996. - Nouvelles données relatives à la séquence de bioindication de l'eutrophisation dans les cours d'eau faiblement minéralisés des Vosges du Nord. Acta bot. Gallica, $142(6): 627-638$.

Thiébaut G. 1997. — Effets des perturbations liées à l'eutrophisation et l'acidification des cours d'eau des Vosges du Nord sur les phytocénoses aquatiques. Approche spatio-temporelle et expérimentale. Thèse Doctorat Univ. de Metz : 208 p. + annexes.

Trémolières M., Carbiener R., Ortscheidt A. \& Klein J-P. 1994. Changes in aquatic vegetation in Rhine floodplain streams in Alsace in relation to disturbance. J. Veg. Sci., $5: 169-178$.

Trémolières M., Roeck U., Klein J.-P.\& Carbiener R. 1993. - The exchange process between river and groundwater on the Central Alsace floodplain (Eastern France). I. The case of a river with functional floodplain. Hydrobiologia, 273 : 19-36.

Wiegleb G. 1981. - Application of multiple discriminant analysis on the analysis of the correlation between macrophyte vegetation and water quality in running waters of Central Europe. Hydrobiologia, 79 : 91-100. 
